\title{
REDES DE INOVAÇÃO: ALINHAMENTO CONCEITUAL E O PANORAMA ATUAL DA TEMÁTICA NO TERRITÓRIO BRASILEIRO
}

\author{
Danielle Nunes Ramos ${ }^{1}$ \\ Clarissa Stefani Teixeira ${ }^{2}$ \\ ${ }^{1}$ Universidade Federal de Santa Catarina - UFSC, Departamento de Ciências Contábeis - VIA Estação \\ Conhecimento \\ ${ }^{2}$ Universidade Federal de Santa Catarina - UFSC \\ Departamento de Engenharia do Conhecimento - Programa de Pós-Graduação em Engenharia e Gestão do \\ Conhecimento - VIA Estação Conhecimento \\ nunesrdanielle@gmail.com \\ clastefani@gmail.com
}

\begin{abstract}
Resumo
O presente estudo buscou analisar as redes com foco em inovação que surgem como uma forma inédita de organização do trabalho e interação entre organizações. As redes foram identificadas por meio de um mapeamento realizado na internet. No Brasil constatou-se a existência de 30 redes e destas, 09 são de inovação. A região Sudeste destaca-se pelo número de redes com (04). Em suma as redes possuem propósitos semelhantes. A interação entre empresários locais, criação de cultura voltada para a importância da inovação e o fortalecimento dos programas de pós-graduação geram a sustentabilidade econômica e consequentemente o fortalecimento dos associados.
\end{abstract}

Palavras-chave: Redes. Inovação. Interação.

\begin{abstract}
The present study aimed to analyze the networks with a focus on innovation that arise as a new form of work organization and interaction between organizations. The nets were identified by means of a mapping done on the internet. In Brazil it was noted the existence of 30 networks and of these, 09 are of innovation. The Southeast region is distinguished by the number of networks with (04). In short the networks have similar purposes. The interaction between local entrepreneurs, creating culture toward the importance of innovation and the strengthening of graduate programs generate economic sustainability and consequently the strengthening of associates.
\end{abstract}

Keywords: Networks. Innovation. Interaction.

\section{Introdução}

Grande volume de informações é veiculado livremente por diversos meios e concomitantemente a isso mudanças vão surgindo na sociedade do conhecimento levando as organizações refletir sobre quais estratégias devem ser adotadas para sobreviverem com sucesso no mundo competitivo no qual a informação e o conhecimento são os bens de maior valor (Rossetti et al 2008). Estudos do Instituto de Pesquisa Econômica Aplicada (IPEA) revelaram que a competitividade da economia brasileira depende diretamente das vantagens adquiridas a

R. Eletr. do Alto Vale do Itajaí - REAVI, v. 5, n. 8, p. 01-13, dez., 2016

ISSN: 2316-4190, DOI: 10.5965/2316419005082016103 
partir da capacitação tecnológica das empresas e do incentivo dado pelos sistemas de inovação (IPEA 2007).

Inovações ajudam a modificar a história da humanidade desde sempre. Do machado às terapias com células-tronco, um conjunto infindável de produtos e de processos modificou as formas de vida (IPEA 2008) e Baumgarten (2008) afirma que a temática das relações entre ciência, tecnologia e sustentabilidade vem assumindo importância crescente no atual mundo globalizado. Em contrapartida, Balestrin, Verschoore e Reyes Júnior (2010) apontam que apenas nos últimos anos é que houve aumento da quantidade e qualidade das pesquisas e publicações que estudam o tema, especialmente no âmbito da inovação.

A inovação não só requer a abertura de novos mercados, mas também exige a implementação de novas formas de servir àqueles já estabelecidos e maduros (Bessant e Tidd 2009). Conforme estabelece o Manual de Oslo, inovação é:

[...] implementação de um produto (bem ou serviço) novo ou significativamente melhorado, ou um processo, ou um novo método de marketing, ou um novo método organizacional nas práticas de negócios, na organização do local de trabalho ou nas relações externas (OCDE 2005).

Já Nascimento e Junior (SN) descrevem que num mundo pós-globalizado, o desenvolvimento econômico, social, cultural e ambiental está pautado em itens como conhecimento, espírito empreendedor, cooperação e redes de contato e que tais fatores possibilitam aos empreendedores modificarem os rumos das relações sociais, por meio do desenvolvimento das empresas de acordo com o novo paradigma, sustentado por conhecimento e inovação, que produz maior valor agregado à sociedade.

As empresas estão se organizando diferente em relação às épocas passadas, fato este que altera o panorama atual, haja vista as exigências em relação à qualidade, redução de custos, inovação e outros aspectos que expõem que as estruturas tradicionais não mais viabilizam as empresas atenderem a essas novas exigências. O progresso de estratégias e estruturas em rede está substituindo as formas organizacionais retrógadas (Verschoore e Balestrin 2008).

As estruturas em rede facilitam que o conhecimento seja disseminado por produtores, fornecedores e usuários localizados em diferentes organizações e facilitam a instantânea troca de informação e os processos decisórios conjuntos (Kuppers e Pyka, 2002). Entretanto, ainda são poucos os estudos que buscam evidenciar a realidade de países e suas ações nas estratégias de formação de redes entre organizações em prol de temas como a inovação e a tecnologia, por exemplo. Dados acerca da atuação das redes são importantes, pois segundo Lash (1994) parte relevante do esforço de inovação ocorre por meio de redes interorganizacionais. Neste contexto, o presente artigo busca analisar as redes com foco em inovação que surgem como uma forma inédita de organização do trabalho e interação entre organizações.

\section{Desenvolvimento}

\subsection{A temática inovação versus redes e redes de cooperação}

Para Katz et al (2000) trabalhar em rede é um processo que organiza e mantém colaborações eficientes. Os objetivos de um trabalho em rede permeiam: acessar ideias, tecnologias e compartilhar informações, experiências, conhecimentos técnicos e negócios entre organizações, 
institutos ou centros de pesquisa tecnológica e universidades. Aquelas redes que mantêm relações interorganizacionais com diversas empresas demonstram opções diferentes de soluções das formas tradicionais para os problemas, fato este facilitador da realização de ações conjuntas e a transação de recursos para obtenção de um objetivo organizacional (Balestrin et al 2010).

Os autores Storper e Harrison (1991) classificam as redes estabelecidas, conforme o grau de hierarquia, em quatro formas básicas, conforme o Quadro 1.

Quadro 1 - Classificação das redes a partir do grau de hierarquia.

\begin{tabular}{|l|l|}
\hline $\begin{array}{l}\text { Redes simétricas ou } \\
\text { flexíveis }\end{array}$ & $\begin{array}{l}\text { Igualitárias na relação entre os integrantes, sem qualquer } \\
\text { espécie de hierarquia entre eles. }\end{array}$ \\
\hline $\begin{array}{l}\text { Redes levemente } \\
\text { assimétricas com } \\
\text { coordenação }\end{array}$ & $\begin{array}{l}\text { Apresentam leve grau de hierarquia devido à relativa } \\
\text { influência da empresa coordenadora, porém limitada e não } \\
\text { determinante da sobrevivência das empresas participantes } \\
\text { do sistema. }\end{array}$ \\
\hline $\begin{array}{l}\text { Redes assimétricas } \\
\text { com empresa líder }\end{array}$ & $\begin{array}{l}\text { Apresentam forte assimetria hierárquica entre a empresa } \\
\text { líder e os integrantes, cuja sobrevivência condiciona-se à } \\
\text { estratégia da líder. }\end{array}$ \\
\hline Redes Hierárquicas & $\begin{array}{l}\text { Estruturadas por meio da plena formalidade entre a empresa } \\
\text { líder e as demais integrantes. }\end{array}$ \\
\hline
\end{tabular}

Fonte: Storper e Harrison (1991)

De acordo com o Quadro 2, Fusco et al (2005) descrevem seis formas de organização baseadas no grau de formalização legal estabelecido entre os integrantes, são elas:

Quadro 2 - Formas de organização das redes.

\begin{tabular}{|l|l|}
\hline $\begin{array}{l}\text { Redes Sociais } \\
\text { Simétricas }\end{array}$ & $\begin{array}{l}\text { Não existem instrumentos legais, tal como um contrato, que } \\
\text { vincule os integrantes; um integrante tem autonomia para aderir } \\
\text { ou desligar-se da rede a qualquer momento. Todos os integrantes } \\
\text { possuem o mesmo poder de tomada de decisão, formando um } \\
\text { agrupamento de caráter essencialmente social. }\end{array}$ \\
\hline $\begin{array}{l}\text { Redes Sociais } \\
\text { Assimétricas }\end{array}$ & $\begin{array}{l}\text { Os integrantes definem sua parceria por meio de contratos que } \\
\text { regularizam a troca de bens e serviços, mas ainda há a liberdade } \\
\text { de um integrante aderir ou desligar-se da rede a qualquer } \\
\text { momento. }\end{array}$ \\
\hline $\begin{array}{l}\text { Redes } \\
\text { Burocráticas }\end{array}$ & $\begin{array}{l}\text { São reguladas através de contratos formais com cláusulas que } \\
\text { estabelecem relações, direitos, obrigações, formalização de trocas } \\
\text { e acordos e proteção dos direitos individuais dos integrantes. }\end{array}$ \\
\hline $\begin{array}{l}\text { Redes } \\
\text { Burocráticas } \\
\text { Assimétricas }\end{array}$ & $\begin{array}{l}\text { Possui instrumentos legais voltados ao alinhamento estratégico } \\
\text { dos integrantes aos interesses do agente gestor, com } \\
\text { estabelecimento de direitos, transferência de conhecimento e } \\
\text { padronização de serviços e informações entre os participantes. }\end{array}$ \\
\hline $\begin{array}{l}\text { Redes } \\
\text { Proprietárias }\end{array}$ & $\begin{array}{l}\text { As empresas integrantes estabelecem uma nova empresa como } \\
\text { forma de equilíbrio de conhecimentos e capitais. Os acordos e } \\
\text { direitos à propriedade dos integrantes são estabelecidos por } \\
\text { contratos formais e cláusulas específicas. }\end{array}$ \\
\hline $\begin{array}{l}\text { Redes } \\
\text { Proprietárias } \\
\text { Assimétricas }\end{array}$ & $\begin{array}{l}\text { Há "risco" envolvido, adicionalmente às características presentes } \\
\text { nas redes Proprietárias Simétricas. Nas Redes Proprietárias } \\
\text { Assimétricas, tem um investidor e a empresa como parceiros de } \\
\text { risco. }\end{array}$ \\
\hline
\end{tabular}

Fonte: Fusco et al (2005) 
Conforme os autores Matos e Afsarmanesh (2008) as interações realizadas nas redes são muitas e estas podem se configurar de forma diferenciada. Os mesmos autores indicam essas diferenças assim como mostra o Quadro 3:

Quadro 3 - Os diferentes modelos de redes existentes.

\begin{tabular}{|l|l|}
\hline $\begin{array}{l}\text { Rede } \\
\text { organizações } \\
\text { (networking) }\end{array}$ & $\begin{array}{l}\text { Fundamentadas nas interações de comunicação e troca de } \\
\text { informações entre organizações para uso individual. Pode } \\
\text { acontecer mesmo objetivos comuns entre os membros. }\end{array}$ \\
\hline Rede coordenada & $\begin{array}{l}\text { A princípio não há objetivos específicos comuns, porém há } \\
\text { coordenação do compartilhamento de informações para que seja } \\
\text { possível o alinhamento dessas atividades de modo a se obter } \\
\text { resultados mais eficientes. }\end{array}$ \\
\hline Cooperação & $\begin{array}{l}\text { É a divisão de atividades específicas entre os indivíduos } \\
\text { participantes. Há a troca de informações e redirecionamento das } \\
\text { tarefas como nos casos anteriores, mas inclui o compartilhamento } \\
\text { de recursos para atingir as metas estabelecidas. }\end{array}$ \\
\hline Colaboração & $\begin{array}{l}\text { Os parceiros trabalham juntos para planejar, implementar e } \\
\text { avaliar os processos interorganizacionais que alinham os } \\
\text { princípios e métodos para compartilhamento de informações e } \\
\text { recursos de modo a se atingir objetivos comuns e } \\
\text { simultaneamente fortalecer as capacidades individuais de cada } \\
\text { parceiro. Atua como uma identidade original onde os riscos, } \\
\text { recursos e responsabilidades são divididos com a intenção de que } \\
\text { os benefícios que motivam a criação da rede de colaboração } \\
\text { sejam alcançados. }\end{array}$ \\
\hline
\end{tabular}

Fonte: Matos e Afsarmanesh (2008)

\section{Metodologia}

Esta pesquisa é classificada como descritiva, pelo fato de descrever as redes brasileiras com enfoque naquelas que apresentam enfoque em inovação e tecnologia (Duarte 2014). Conforme Vergara (2000) a pesquisa descritiva desvenda características de determinada população ou fenômeno, por meio de um levantamento ou observações de suas características.

Além disso, a abordagem utilizada no presente estudo tem viés qualitativo, pois segundo Godoy (1995, p. 63):

Quando o estudo é de caráter descritivo e o que se busca é o entendimento do fenômeno como um todo, na sua complexidade, é possível que uma análise qualitativa seja a mais indicada. Ainda quando a nossa preocupação for a compreensão da teia de relações sociais e culturais que se estabelecem no interior das organizações, o trabalho qualitativo pode oferecer interessantes e relevantes dados.

Ou seja, quando a pesquisa busca um entendimento do contexto como um todo, é mais interessante utilizar-se da pesquisa qualitativa, pois esta faz uma análise mais profunda.

O presente estudo foi realizado em três fases, sendo: i) identificação das redes, ii) localização de documentação pertinente a análise, iii) identificação da abrangência e foco de cada rede.

As fases da pesquisa podem ser assim definidas: 
- Fase 1 - Identificação das redes brasileiras

Inicialmente foram mapeadas as redes com foco em inovação. As buscas foram realizadas em todos os estados brasileiros. Para tanto, foram realizadas buscas em base de dados utilizando os seguintes termos: rede inovação, rede tecnologia. Para o rastreamento das redes todos os estados brasileiros foram combinados, como por exemplo: "rede inovação" "Rio Grande do Sul". Assim, serão realizadas buscas com todos os termos citados nos 11 estados brasileiros e Distrito Federal.

- Fase 2 - Localização de documentação pertinente à análise

Após o mapeamento das redes por estado foram localizadas documentações pertinentes para o desenvolvimento do estudo. Como exemplo de documentações, além de artigos foram localizados os sites de cada rede e seus respectivos estatutos, uma vez que estes definem as regras e o funcionamento das organizações.

- Fase 3 - Identificação da abrangência e foco de cada rede

Nesta fase foram identificados os quantitativos como o tempo de existência de cada rede, a territorialização, o número de associados e o propósito de cada rede. Estes dados foram importantes para a definição da abrangência de cada uma das redes.

\section{Resultados e discussão}

\subsection{A realidade das redes no território brasileiro}

Este estudo buscou realizar uma análise sobre as redes brasileiras com enfoque em inovação, estas facilitam e potencializam as ações conjuntas para atingir objetivos específicos traçados em âmbito organizacional. Constatou-se que no Brasil há 30 redes em funcionamento, sendo que considerando a temática de inovação há 09 redes em funcionamento nas diferentes regiões. $\mathrm{O}$ Quadro 4 ilustra as redes brasileiras com enfoque em inovação.

\begin{tabular}{|l|l|l|}
\multicolumn{3}{|c|}{ Quadro 4 - Redes de inovação brasileiras } \\
\hline Região & Estado & Nome da Rede \\
\hline Norte & Pará & Redes - Inovação e Sustentabilidade econômica \\
\hline $\begin{array}{l}\text { Centro- } \\
\text { Oeste }\end{array}$ & Brasília & $\begin{array}{l}\text { Rede Centro Oeste de Pós-Graduação, Pesquisa e } \\
\text { Inovação - Pró-Centro-Oeste }\end{array}$ \\
\hline \multirow{4}{*}{ Sudeste } & São Paulo & Rede Paulista de Inovação em governo \\
\cline { 2 - 3 } & Rio de Janeiro & Rede de Tecnologia e Inovação do Rio de Janeiro \\
\cline { 2 - 3 } & Minas Gerais & Rede Mineira de Inovação \\
\cline { 2 - 3 } & Minas Gerais & Rede de Inovação de Itajubá \\
\hline \multirow{3}{*}{ Sul } & Paraná & Rede Paranaense de Tecnologia e Inovação \\
\cline { 2 - 3 } & Paraná & Rede de Inovação \\
\cline { 2 - 3 } & Santa Catarina & Rede Catarinense de Inovação \\
\hline
\end{tabular}

Considerando a abrangência das redes em nível territorial, observa-se que na região Nordeste do país não há redes com enfoque específico em inovação, diferentemente das demais regiões, pois todas apresentam, no mínimo, 01 e, no máximo, 04 redes por região - com destaque na região Sudeste.

As redes são presentes em diversos países. Segundo a Comissão Europeia (2004) mais da metade das pequenas e médias empresas apresentam práticas de cooperação - formais ou informais. Os países de destaque são Finlândia, Islândia, Dinamarca e Itália. Na Itália há iniciativas políticas que motivam o uso de práticas de redes, destacam-se: acesso a novos e 
maiores mercados, mais amplo fornecimento de produtos, e acesso a know-how e tecnologia, entre outros.

Autores como Prahalad e Ramaswamy (2004) indicam que o lócus da inovação deve estar em um ambiente de experimentação em rede que não fica restrito apenas as cadeias produtivas, mas sim as redes de competências. Desta forma, ao se analisar as redes brasileiras encontradas observa-se que as instituições que compõe a interação de cada rede são diversas. Grande parte das redes apresentam instituições como universidades, centros de pesquisa, escolas técnicas, instituições de fomento, Serviço Brasileiro de Apoio a Micro e Pequena Empresa (SEBRAE) e Conselho Nacional de Desenvolvimento Científico e Tecnológico (CNPq) como parceiros. O fato do número de conexões realizadas pela rede, por meio do número de entidades ligadas a ela - associadas - pode fazer o diferencial em termos de competitividade (Verschoore e Balestrin 2008). O Quadro 5 ilustra o número de associados em cada uma das redes com foco em inovação.

Quadro 5 - Número de associados às redes de inovação do Brasil.

\begin{tabular}{|l|c|}
\hline \multicolumn{1}{|c|}{ Redes com foco em Inovação } & $\begin{array}{c}\text { Número de } \\
\text { associados }\end{array}$ \\
\hline Redes - Inovação e Sustentabilidade econômica & - \\
\hline $\begin{array}{l}\text { Rede Centro Oeste de Pós-Graduação, Pesquisa e Inovação - Pró- } \\
\text { Centro-Oeste }\end{array}$ & - \\
\hline Rede Paulista de Inovação em governo & - \\
\hline Rede de Tecnologia e Inovação do Rio de Janeiro & 53 \\
\hline Rede Mineira de Inovação & 30 \\
\hline Rede de inovação de Itajubá & 03 \\
\hline Rede Paranaense de Tecnologia e Inovação & 14 \\
\hline Rede de Inovação & 04 \\
\hline Rede Catarinense de Inovação & 140 \\
\hline
\end{tabular}

Entretanto as redes Inovação e Sustentabilidade econômica, Rede Centro Oeste de PósGraduação, Pesquisa e Inovação - Pró Centro-Oeste e Rede Paulista de Inovação em Governo não apresentam o número de associados em suas home pages, o que representa aproximadamente $33,33 \%$ de todas as redes com a temática de inovação existentes no Brasil. O destaque das redes, para o número de associados está na Rede Catarinense de Inovação com 140 associados. A abrangência da rede também é ponto importante. No caso do presente estudo, algumas redes não se limitam ao município ou estado que elas estão alocadas (física ou virtualmente).

Os enfoques das redes, nas diferentes regiões e até nos diferentes estados podem ser observadas no Quadro 6.

Quadro 6 - Proposta das redes de Inovação e/ou Tecnológica. Redes - Inovação e Sustentabilidade econômica Atender as demandas das indústrias locais com geração de interação entre os empresários paraenses e as empresas que fazem os maiores investimentos no estado, basicamente age como uma rede integradora de agentes estratégicos, para promover a sustentabilidade econômica no ambiente de negócios da Amazônia Legal (REDES - INOVAÇÃO E SUSTENTABILIDADE ECONÔMICA, 2015).

Rede Centro Oeste de Pós-Graduação, Pesquisa e Inovação - Pró-Centro-Oeste Apoio à pesquisa em biotecnologia e biodiversidade na Região Centro-Oeste, consolidar e 
integrar grupos de pesquisa da região, formar, atrair e fixar doutores na região, fortalecer e contribuir para a consolidação de programas de pós-graduação, instituir um programa de doutorado de caráter multi-institucional e contribuir para o desenvolvimento sustentável da região. (REDE CENTRO OESTE DE PÓS-GRADUAÇÃO, PESQUISA E INOVAÇÃO PRÓ-CENTRO-OESTE, 2015).

\section{Rede Paulista de Inovação em governo}

Busca a melhoria da eficiência, eficácia, efetividade e qualidade da formulação e implantação de políticas públicas e serviços ao cidadão e à sociedade; promoção da transparência na gestão pública por meio do provimento de informações governamentais ao cidadão; incentivo à criação de cultura voltada para a importância da inovação e da geração e compartilhamento de conhecimento e informação na gestão pública, entre os dirigentes governamentais; o desenvolvimento de cultura colaborativa e inovadora intra e intergovernamental, com a geração e compartilhamento de conhecimento e informações entre áreas governamentais e entre governo e sociedade; a promoção de oportunidades de aprendizado contínuo aos servidores; a promoção da adoção e capacitação dos servidores na adoção de ferramentas de informática e uso da Internet para fins da Gestão do Conhecimento e Inovação e a divulgação dos resultados e benefícios da implantação da Política de Gestão do Conhecimento e Inovação (REDE PAULISTA DE INOVAÇÃO EM GOVERNO, 2015).

\section{Rede de Tecnologia e Inovação do Rio de Janeiro}

Estímulo, fomento, apoio e mobilização aos diversos segmentos da sociedade e dos poderes públicos, em toda e qualquer atividade, que tenha como objetivo promover a pesquisa, $\mathrm{o}$ desenvolvimento e a implantação de inovações tecnológicas, científicas e culturais realizadas no Rio de Janeiro (REDE DE TECNOLOGIA E INOVAÇÃO DO RIO DE JANEIRO, 2015).

\section{Rede Mineira de Inovação}

Integrar a pesquisa, a tecnologia e a inovação para que, juntas, propiciem negócios competitivos e, consequentemente, o fortalecimento de seus associados (REDE MINEIRA DE INOVAÇÃO, 2015).

\section{Rede de inovação de Itajubá}

Incentivo à inovação na cidade de Itajubá e as cidades vizinhas tendo em vista o fortalecimento dos instrumentos de inovação tecnológica e facilitação da interação entre empresários, pesquisadores, membros do setor público e estudantes fazendo atividades interdependentes e complementares entre os atores dos processos de inovação (REDE DE INOVAÇÃO DE ITAJUBÁ, 2015).

\section{Rede Paranaense de Tecnologia e Inovação}

Execução, promoção, fomento e apoio às atividades atreladas a educação, desenvolvimento institucional, inovação e desenvolvimento científico e tecnológico e aplicação na criação e/ou desenvolvimento de empresas e empreendimentos de base tecnológica, bem como atividades de gestão e transferência de tecnologias e promoção do capital humano (REDE PARANAENSE DE TECNOLOGIA E INOVAÇÃO, 2015).

\section{Rede de Inovação}

Transferência de conhecimento, experiências e boas práticas de inovação para empreendedores e criação de valor para as empresas associadas (REDE DE INOVAÇÃO, 2015).

\section{Rede Catarinense de Inovação}

Integra esforços para ampliar o número de incubadoras nas diferentes regiões do estado e, ao mesmo tempo, contribuir para a consolidação daquelas que estão em funcionamento e visa também reunir entidades públicas e privadas com interesses comuns ao propósito e 
proporcionar integração com incubadoras, parques tecnológicos, distritos de inovação, núcleos de inovação tecnológica e outros atores. (REDE CATARINENSE DE INOVAÇÃO, 2015).

Em suma as redes possuem propósitos parecidos. A interação entre empresários locais, criação de cultura voltada para a importância da inovação e o fortalecimento dos programas de pós-graduação geram a sustentabilidade econômica e consequentemente o fortalecimento dos associados, finalidades estas apresentadas em quase todas as 08 redes existentes com a temática da presente análise.

Tálamo (2008) afirma que para enfrentar o ambiente de incerteza, as organizações investiram na criação de várias modalidades de agrupamentos empresariais entre elas diversas modalidades de rede. Estas, de acordo com Silva (2006) vêm recebendo no Brasil destaque em estudos na área universitária e conta com o apoio do setor público. Além disso, para Todeva (2006) a atuação em rede torna provável a realização de ações conjuntas e a transação de recursos para que os objetivos organizacionais sejam alcançados.

Conforme Malafaia et al (2007) o estabelecimento do capital social, gerado pela troca de conveniências, é o principio para que se tenha relações de confiança, fator este fundamental ao desenvolvimento conjunto. Os autores afirmam ainda que a confiança é barreira e caminho simultaneamente e que a construção da confiança mútua é a grande barreira à formação de redes de cooperação, ao mesmo tempo em que é fundamental ao êxito da rede.

\section{Considerações finais}

O presente estudo buscou apresentar o trabalho das redes de cooperação e classificação das mesmas conforme grau de hierarquia e grau de formalização legal e os diferentes modelos de redes de cooperação existentes.

No Brasil constatou-se a existência de 30 redes e destas, 09 são de inovação e cada uma teve seus objetivos analisados e descritos, por região. A região Sudeste destaca-se pelo número de redes com este viés (04) seguida da região Sul que possui 03, Norte e Centro-oeste (01). A região Nordeste não apresentou nenhuma rede neste sentido. De maneira geral, pode-se dizer que o propósito das redes de inovação no Brasil apresenta semelhanças. A interação entre empresários locais, criação de cultura voltada para a importância da inovação e o fortalecimento dos programas de pós-graduação geram a sustentabilidade econômica e consequentemente o fortalecimento dos associados, finalidades estas apresentadas em quase todas as 09 redes existentes com a temática da presente análise.

\section{Referências Bibliográficas}

Balestrin, A.; Verschoore, J. R.; Reyes Júnior, E (2010). O campo de estudo sobre redes de cooperação interorganizacional no Brasil. Revista de Administração Científica, v. 14, n. 3, p. 458-477.

Baumgarten, M. (2008). Ciência, tecnologia e desenvolvimento - redes e inovação social. Revista Parcerias Estratégicas, n 26

Bessant, J.; Tidd, J. Inovação e empreendedorismo: administração. Porto Alegre, Bookman Editora, $512 \mathrm{p}$.

Camarinha-Matos, L. M., Afsarmanesh, H. (Eds) (2008). Collaborative Networks: reference modeling. New York: Springer 
De Negri, J. A. (2007). "Relatório de pesquisa sobre inovação tecnológica nas empresas industriais brasileiras". Brasília: Ipea

De Negri, J. A.; Kubota L. C. (2008). "Políticas de Incentivo à Inovação Tecnológica no Brasil”. Brasília: Ipea

European Commission. Observatory of European SMEs 2003, No. 5 - SMEs and cooperation. 2004 a.

European Commission. Observatory of European SMEs 2003, No. 8 - Highlights from the 2003 Observatory. 2004 b.

Fusco, J. P. A. et AL (2005). Redes produtivas e cadeias de fornecimento. São Paulo: Arte \& Ciência

Katz, R. et al. Organizations. In: DORF, Richard C. (Ed.). The technology management handbook. Boca Raton: CRC Press, 2000. Cap. 7.

Kuppers, G.;Pyka, A. (2002). The self-organization of innovation networks: introductory remarks in innovation networks. Theory and practice, Cheltenham: Edward Elgar

Lash, S. (1994). Reflexivity and its doubles: structure, aesthetics and community. In U. Beck, A. Giddens, \& S. Lash (Orgs.). Reflexive Modernization. Londres: Polity Press

Malafaia, G. C. et al. (2007) capital social e a construção da confiança em redes de cooperação: mudando padrões de relacionamento na pecuária de corte. In: ENCONTRO DA ANPAD, 31.,2007. Anais... Rio de Janeiro: ANPAD

Martes, A. C. B.; Bulgacov, S.; Nascimento, M. R. do; Gonçalves, S. A.; Augusto, P. M. (2006). "Fórum - redes sociais e interorganizacionais". Revista de Administração de Empresas, v. 46, n. 3, p. 1015

Nascimento, D. E. do, Junior, S. L. Ambientes e dinâmica de cooperação para Inovação. Aymará Educação, 120 p.

OCDE. Manual de Oslo. (2005) Diretrizes para coleta e interpretação de dados sobre inovação. 3. ed. Brasília: Finep

Redes - Inovação e Sustentatibilidade Econômica, 2015. Disponível em:

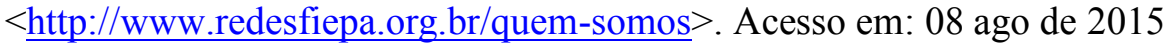

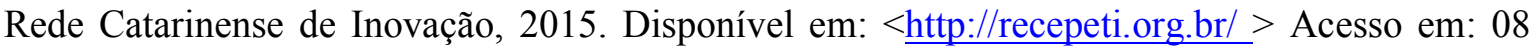
ago de 2015

Rede Centro Oeste de Pós-graduação, Pesquisa e Inovação - Pró-Centro-Oeste, 2015. Disponível

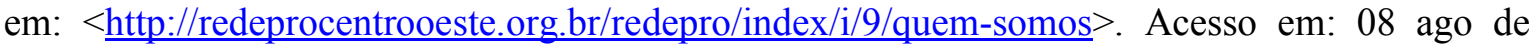
2015

Rede de Inovação de Itajubá, 2015. Disponível em: <http://inovacaoitajuba.com.br/>. Acesso em: 08 ago de 2015 
Rede de Inovação, 2015.2015 Disponível <http://www.redeinovacao.org.br/Paginas/SobreaRededeInov>. Acesso em: 08 ago de 2015

Rede de Tecnologia e Inovação do Rio de Janeiro, 2015. Disponível em: $<\underline{\text { http://www.redetec.org.br/> }}$. Acesso em: 08 ago de 2015

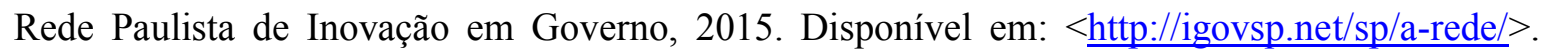
Acesso em: 08 ago de 2015

Rede Mineira de Inovação, 2015. Disponível em: <http://www.rmi.org.br/rmi/Home>. Acesso em: 08 ago de 2015

Rede Paranaense de Tecnologia e Inovação, 2015. Disponível em: < $\underline{\text { http://www.reparte.org.br/>. }}$ Acesso em: 08 ago de 2015

Rossetti, A.; Pacheco A. P. R.; Salles, B.; Garcia, M.; Santos, N. (2009). "A organização baseada no conhecimento: novas estruturas, estratégias e redes de relacionamento". Ciência da Informação, número 1, Brasília

Silva, C. A. V. Redes de cooperação no Brasil e no mundo: uma abordagem reflexiva In: EGEPE - ENCONTRO DE ESTUDOS SOBRE EMPREENDEDORISMO E GESTÃO DE PEQUENAS EMPRESAS. 4. 2005, Curitiba, Anais... Curitiba, 2005, p. 1279-1288

Storper, M.; Harrison, B. (1991). Flexibility, hierarchy and regional developments: the changing structure of industrial production systems and their forms of governance in the 1990s. Research Policy, v. 20, n. 5

Todeva, E. (2006). Business networks. Strategy and structure. London: Routledge 